\title{
Photoinduced microchannels inside silicon by femtosecond pulses
}

$\operatorname{AUTHOR}(\mathrm{S}):$

Chen, Tao; Si, Jinhai; Hou, Xun; Kanehira, Shingo; Miura, Kiyotaka; Hirao, Kazuyuki

\section{CITATION:}

Chen, Tao ...[et al]. Photoinduced microchannels inside silicon by femtosecond pulses. APPLIED PHYSICS LETTERS 2008, 93(5): 051112.

\section{ISSUE DATE:}

2008-08-04

URL:

http://hdl.handle.net/2433/84593

\section{RIGHT:}

Copyright 2008 American Institute of Physics. This article may be downloaded for personal use only. Any other use requires prior permission of the author and the American Institute of Physics. 


\title{
Photoinduced microchannels inside silicon by femtosecond pulses
}

\author{
Tao Chen, ${ }^{1}$ Jinhai $\mathrm{Si},{ }^{1, a)}$ Xun Hou, ${ }^{1}$ Shingo Kanehira, ${ }^{2}$ Kiyotaka Miura, ${ }^{2}$ and \\ Kazuyuki Hirao ${ }^{2}$ \\ ${ }^{1}$ Key Laboratory for Physical Electronics and Devices of the Ministry of Education and Shaanxi Key \\ Laboratory of Information Photonic Technique, School of Electronics and information Engineering, Xi'an \\ Jiaotong University, Xianning-xilu 28, Xi'an 710049, China \\ ${ }^{2}$ Department of Material Chemistry, Kyoto University, Nishikyo-ku, Kyoto 615-8510, Japan
}

(Received 30 May 2008; accepted 21 July 2008; published online 7 August 2008)

\begin{abstract}
We reported on the fabrication of microchannels in the interior of silicon wafers using a femtosecond laser of $800 \mathrm{~nm}$ wavelength, which was in the absorption region of silicon. The scanning electron micrographs showed that microchannels were induced inside the silicon wafer when the femtosecond laser beam was focused inside the wafer. The aspect ratio of the microchannel cross section decreased with the increase in scan velocity of the laser. The formation of the photoinduced microchannels probably resulted from the microexplosions due to both the linear absorption and avalanche ionization. () 2008 American Institute of Physics.
\end{abstract}

[DOI: $10.1063 / 1.2969401]$

The interaction of ultrashort lasers with silicon has attracted considerable attention due to its potential use in microelectromechanism system devices and silicon-based optoelectronic devices. The microstructure formed on the silicon surface under the irradiation of femtosecond lasers has been widely studied in vacuum, water, and gases. Femtosecond laser ablation of silicon targets in vacuum has been shown to be a viable route to the generation and deposition of nanoparticles. ${ }^{1}$ In the presence of $\mathrm{SF}_{6}$, the femtosecond laser induced conical spikes on the silicon surface. ${ }^{2}$ Photodetectors fabricated with such silicon exhibit enhanced nearinfrared response. ${ }^{3}$ The microfabrication on silicon surfaces in air by femtosecond laser pulses can improve the silicon's light emission efficiency. ${ }^{4}$ In addition, the submicrometer silicon spikes were induced in water. ${ }^{5}$

The microfabrication using femtosecond lasers has been performed on surfaces and in the interior of glasses, ${ }^{6-11}$ polymers, ${ }^{12-14}$ and hybrid inorganic-organic materials. ${ }^{15}$ However, the majority of studies on the microfabrication of silicon using femtosecond lasers are conducted on the silicon surface. It is probably because that silicon is opaque to the $800 \mathrm{~nm}$ laser light provided by most femtosecond laser amplifiers, or the microstructures formed inside silicon could not be easily observed as that inside transparent materials. Recently, Nejadmalayeri et al. demonstrated the buried waveguide induced in silicon by a femtosecond laser at $2.4 \mu \mathrm{m} .{ }^{16}$ This work extends the ultrafast three-dimensional modification technique to silicon. It is expected that the microstructures formed inside silicon can potentially be used in the silicon-based photonic crystals, optical waveguides, microfluidic devices, and so on.

In this letter, we induced the microstructures in the interior of silicon wafers using a femtosecond laser at $800 \mathrm{~nm}$. When the femtosecond laser beam was focused inside the silicon wafer by a small numerical aperture (NA) lens, a $5 \mu \mathrm{m}$ diameter microchannel with circular cross section formed inside the wafer, which was accompanied with surface damage. Because the photon energy of the laser is above

\footnotetext{
a) Author to whom correspondence should be addressed. Electronic mail: jinhaisi@mail.xjtu.edu.cn.
}

the $1.1 \mathrm{eV}$ bandgap of silicon, the photoinduced microchannel should be attributed to the microexplosions due to both the linear absorption and avalanche ionization.

We performed our experiments on 3-mm-thick silicon wafers that were cleaned in an ultrasonic acetone bath, followed by a methanol bath. The laser source was a Ti:sapphire regenerative amplifier (Coherent, RegA9000), which delivered a train of $800 \mathrm{~nm}, 120 \mathrm{fs}$ laser pulses at a repetition rate of $250 \mathrm{kHz}$. The laser beam was focused inside the silicon wafer via a $5 \times$ microscope objective (Nikon, NA $=0.15$ ). The focused spot size of the laser beam was estimated to be $\sim 10 \mu \mathrm{m}$. The sample was placed on a computer controlled three-dimensional translation stage that was mounted on a microscope system. The laser fluence was varied by a variable attenuator. The damage lines with a $100 \mu \mathrm{m}$ period were produced by translating the silicon wafer at a constant velocity in the plane perpendicular to the laser beam. The morphology of the photoinduced microstructures was characterized with scanning electron microscopy (SEM). The silicon surface perpendicular to the scan direction was polished to observe the channels inside the silicon wafer.

First, the laser beam was focused at $\sim 20 \mu \mathrm{m}$ below the silicon surface. The scan velocity of the laser and the laser fluence were set at $3000 \mu \mathrm{m} / \mathrm{s}$ and $0.45 \mathrm{~J} / \mathrm{cm}^{2}$, respectively. After irradiation with the laser pulses, a groove formed on the silicon surface. Figure 1(a) illustrates the top-view SEM micrograph of the photoinduced microstructures on the silicon surface. The ablated debris was deposited on both sides of the groove. Figures 1(b) and 1(c) illustrate the side-view micrographs of the polished side surface, perpendicular to the laser scan direction. From Figs. 1(b) and 1(c), we can see that microchannels with $5 \mu \mathrm{m}$ diameter formed at $\sim 20 \mu \mathrm{m}$ below the surface of the silicon wafer. The interior surface of the microchannels is rough and there is some debris in the microchannels.

Similar microchannels have been fabricated inside transparent materials ${ }^{10,17}$ and the silicon wafers with grown $\mathrm{SiO}_{2}$ films, ${ }^{18}$ in which the microchannels could be induced inside materials by multiphoton absorption although materials are transparent to the $800 \mathrm{~nm}$ femtosecond laser. Usually, the 

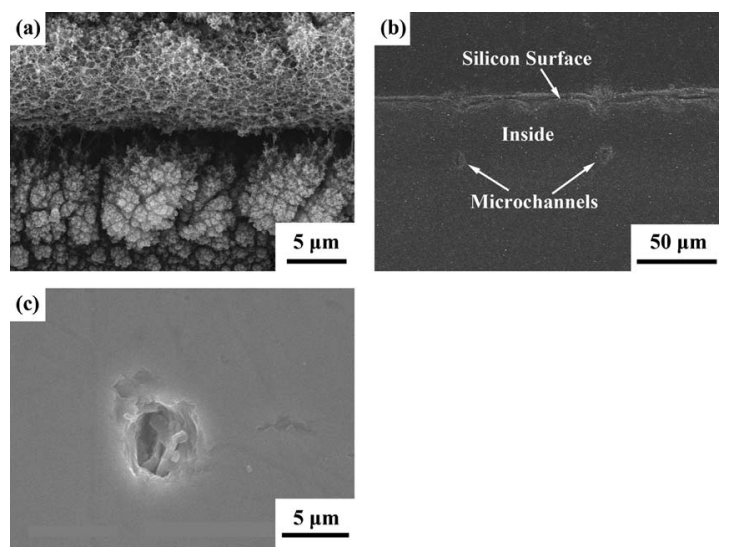

FIG. 1. Scanning electron micrographs of the photoinduced microstructures: (a) top-view micrograph and [(b) and (c)], side-view micrographs. The laser beam was focused at $\sim 20 \mu \mathrm{m}$ below silicon surface. The laser fluence and the scan velocity were set at $0.45 \mathrm{~J} / \mathrm{cm}^{2}$ and $3000 \mu \mathrm{m} / \mathrm{s}$, respectively.

$800 \mathrm{~nm}$ femtosecond laser is only used to induce surface microstructures of silicon due to the linear absorption of silicon at the wavelength of $800 \mathrm{~nm}$. Our results show the possibility of three-dimensional microfabrication inside silicon using $800 \mathrm{~nm}$ femtosecond laser pulses.

In transparent materials as glasses, it is difficult to fabricate circular sectional microchannels by femtosecond laser radiation when samples are translated perpendicularly to the beam propagation direction. ${ }^{6,8}$ Such microchannels could be induced by increasing the NA of the focal lens ${ }^{8}$ or translating the sample along the beam propagation direction. ${ }^{6}$ Our experimental results show that circular sectional microchannels could be easily induced inside silicon even using a microscope objective with NA of 0.15 .

Then, we studied the formation of the microchannel by setting the scan velocity at 1000 and $200 \mu \mathrm{m} / \mathrm{s}$, respectively. The surface damages shown in Fig. 1(a) were observed on both samples. Figure 2 shows the side-view micrographs of the induced microchannels inside the silicon wafers for different scan velocities. From the side-view SEM micrographs, we can see that when the scan velocity was decreased from 3000 to 1000 and $200 \mu \mathrm{m} / \mathrm{s}$, the size of the cross section in the horizontal direction for the photoinduced microchannels increased from 5 to 6 and $7 \mu \mathrm{m}$, respectively, while the size in the vertical direction increased from 5 to 8 and $10 \mu \mathrm{m}$, respectively. With the decrease in the scan velocity, the aspect ratio of the microchannel cross section increased, and the cross sectional shape of the microchannels became ellipse. This is probably because the silicon's linear absorption confined the penetrative depth of the laser pulses. For the slower scan velocity, more laser pulses were accumulated at
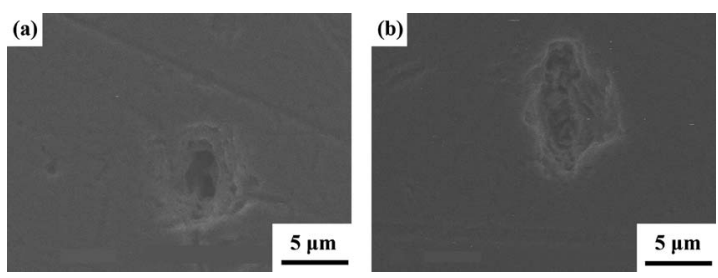

FIG. 2. Side-view scanning electron micrographs of microchannels induced inside silicon. The laser beam was focused at $\sim 20 \mu \mathrm{m}$ below the silicon surface. The laser fluence was $0.45 \mathrm{~J} / \mathrm{cm}^{2}$. The scan velocities were set at (a) $1000 \mu \mathrm{m} / \mathrm{s}$ and (b) $200 \mu \mathrm{m} / \mathrm{s}$, respectively. each position. The first arriving pulses could induce a cavity that could make the following pulses penetrate further into silicon and ablate more silicon in the laser propagation direction.

In our experiments, the microchannels shown above were observed by polishing the sample to a random position. Therefore, we believe that the microchannels have a good level of continuity. Usually, the ablated debris that is redeposited in the microchannels might cause a block in the channels, as that in glasses. We expect that the ablated debris in the microchannels could be removed by water and other solvents assisted ablation with femtosecond laser pulses. ${ }^{17}$

The formation of the microchannels could be explained with the following microexplosion model. ${ }^{9,19}$ Initially, the laser pulses created a hot, high-density electron plasma in the focal volume, which transferred its excess energy to the lattice. The focal volume was heated to a high temperature. The rise in temperature at a constant volume caused immense pressure, which forced material from the center of the explosion outward. During cooling, the material did not anneal and denser phase was frozen in. Therefore, a cavity, surrounded by a region of compacted material, was formed in the microexplosion, while some debris was deposited in the microchannels.

Usually, the inner structures created in glasses using $800 \mathrm{~nm}$ laser pulses and in silicon using $2.4 \mu \mathrm{m}$ laser pulses result from nonlinear absorption, which may be different from the mechanism of the microchannels induced inside silicon by $800 \mathrm{~nm}$ laser pulses. Because the photon energy associated with the $800 \mathrm{~nm}$ light was above the bandgap of silicon, the microexplosion could be initialed by one-photon absorption. It has been proved that the inner structure can be induced even through the one-photon process. ${ }^{20}$ In addition, the nonlinear processes, such as two-photon absorption ${ }^{21}$ and avalanche ionization, 22 could become dominant in laser absorption processes for ultrafast above-gap radiation in silicon. Therefore, we conclude that the microexplosions due to both the linear absorption and the nonlinear processes should be responsible for the photoinduced microchannel.

In conclusion, we have induced $5 \mu \mathrm{m}$ diameter microchannels with circular cross section in the interior of silicon wafers by $800 \mathrm{~nm}$ femtosecond laser pulses even though the wavelength is in the linear absorption region of silicon. The aspect ratio of the microchannel cross section increases with the decrease in the scan velocity of the laser. The formation of the microchannels can be explained with the microexplosion model. The microexplosions might be driven by both linear and nonlinear processes. Our results have showed the possibility of three-dimensional fabrication inside silicon using $800 \mathrm{~nm}$ femtosecond laser pulses.

The authors gratefully acknowledge the financial support for this work provided by the National Science Foundation of China under the Grant No. 10674107 and the National Key Scientific Research Foundation of China under the Grant No. 2006CB921602. The authors thank Dr. M. Sakakura and M. Shimizu for the help in experiments and Professor Shimotsuma for helpful discussion.

\footnotetext{
${ }^{1}$ S. Amoruso, R. Bruzzese, N. Spinelli, R. Velotta, M. Vitiello, X. Wang, G Ausanio, V. Lannotti, and L. Lanotte, Appl. Phys. Lett. 84, 4502 (2004) ${ }^{2}$ T. H. Her, R. J. Finlay, C. Wu, S. Deliwala, and E. Mazur, Appl. Phys. Lett. 73, 1673 (1998)

${ }^{3}$ Z. Huang, J. E. Carey, M. Liu, X. Guo, E. Mazur, and J. C. Campbell,
} 
Appl. Phys. Lett. 89, 033506 (2006).

${ }^{4}$ C. Wu, C. H. Crouch, L. Zhao, and E. Mazur, Appl. Phys. Lett. 81, 1999 (2002).

${ }^{5}$ M. Y. Shen, C. H. Crouch, J. E. Carey, and E. Mazur, Appl. Phys. Lett. 85, 5694 (2004).

${ }^{6}$ K. M. Davis, K. Miura, N. Sugimoto, and K. Hirao, Opt. Lett. 21, 1729 (1996).

${ }^{7}$ A. Ben-Yakar, R. L. Byer, A. Harkin, J. Ashmore, H. A. Stone, M. Shen, and E. Mazur, Appl. Phys. Lett. 83, 3030 (2003).

${ }^{8}$ C. B. Schaffer, A. O. Jamison, and E. Mazur, Appl. Phys. Lett. 84, 1441 (2004).

${ }^{9}$ S. Juodkazis, H. Misawa, T. Hashimoto, E. G. Gamaly, and B. LutherDavies, Appl. Phys. Lett. 88, 201909 (2006).

${ }^{10}$ V. Maselli, R. Osellame, G. Cerullo, R. Ramponi, P. Laporta, L. Magagnin, and P. L. Cavallotti, Appl. Phys. Lett. 88, 191107 (2006).

${ }^{11}$ S. Kanehira, J. Si, J. Qiu, K. Fujita, and K. Hirao, Nano Lett. 5, 1591 (2005).

${ }^{12}$ S. Kawata, H. Sun, T. Tanaka, and K. Takada, Nature (London) 412, 697 (2001).
${ }^{13}$ J. Si, J. Qiu, J. Zhai, Y. Shen, and K. Hirao, Appl. Phys. Lett. 80, 359 (2002).

${ }^{14}$ M. Straub, M. J. Ventura, and M. Gu, Phys. Rev. Lett. 91, 043901 (2003).

${ }^{15}$ G. Qian, J. Guo, M. Wang, J. Si, J. Qiu, and K. Hirao, Appl. Phys. Lett. 83, 2327 (2003).

${ }^{16}$ A. H. Nejadmalayeri, P. R. Herman, J. Burghoff, M. Will, S. Nolte, and A. Tunnermann, Opt. Lett. 30, 964 (2005).

${ }^{17}$ Y. Li, K. Itoh, W. Watanabe, K. Yamada, D. Kuroda, J. Nishii, and Y. Jiang, Opt. Lett. 26, 1912 (2001).

${ }^{18}$ J. P. McDonald, V. R. Mistry, K. E. Ray, and S. M. Yalisove, Appl. Phys. Lett. 88, 183113 (2006).

${ }^{19}$ E. N. Glezer and E. Mazur, Appl. Phys. Lett. 71, 882 (1997).

${ }^{20}$ X. M. Wei, K. P. Chen, D. Coric, P. R. Herman, and J. Li, Proc. SPIE 4637, 251 (2002)

${ }^{21}$ D. H. Reitze, T. R. Zhang, W. M. Wood, and M. C. Downer, J. Opt. Soc. Am. B 7, 84 (1990).

${ }^{22}$ P. P. Pronko, P. A. VanRompay, C. Horvath, F. Loesel, T. Juhasz, X. Liu, and G. Mourou, Phys. Rev. B 58, 2387 (1998). 